\title{
HIDROCARBONETOS E ESTEROIS COMO INDICADORES DE FONTES E DESTINO DE MATÉRIA ORGÂNICA EM SEDIMENTOS DA BAÍA DE SEPETIBA, RIO DE JANEIRO
}

\author{
Renato S. Carreira*, Patrícia V. Ribeiro e Carlos E. M. Silva \\ Faculdade de Oceanografia, Universidade do Estado do Rio de Janeiro, 20550-013 Rio de Janeiro - RJ, Brasil \\ Cassia O. Farias \\ Departamento de Química, Pontifícia Universidade Católica do Rio de Janeiro, 22453-900 Rio de Janeiro - RJ, Brasil
}

Recebido em 15/9/08; aceito em 11/3/09; publicado na web em 4/8/09

\begin{abstract}
HYDROCARBONS AND STEROLS AS INDICATORS OF SOURCE AND FATE OF ORGANIC MATTER IN SEDIMENTS FROM SEPETIBA BAY, RIO DE JANEIRO. The inputs of organic matter derived from natural and anthropogenic sources to Sepetiba Bay were investigated by using aliphatic hydrocarbons and sterols in superficial sediments. Concentrations ranged from 0.26 to $2.65 \mu \mathrm{g} \mathrm{g}^{-1},<0.01$ to $17.41 \mu \mathrm{g} \mathrm{g}^{-1}$ and 0.77 and $9.24 \mu \mathrm{g} \mathrm{g}^{-1}$ for n-alkanes, UCM (unresolved complex mixture) and total sterols, respectively. The selected markers and ratios among individual compounds showed the major contribution of terrestrial sources to the total pool of sedimentary organic matter in the bay, but the accumulation of autochthonous organic matter increased with distance from the shoreline. The input of petroleum hydrocarbons and sewage (coprostanol in the range 0.01 to $0.43 \mu \mathrm{g} \mathrm{g}^{-1}$ ) were also detected, especially in the more urbanized regions of the bay, but at lower levels of contamination when compared to estuaries in other Brazilian coastal regions.
\end{abstract}

Keywords: Sepetiba Bay; sediments; organic matter.

\section{INTRODUÇÃO}

A matéria orgânica em sedimentos costeiros tem origem em diversas fontes - terrestres, aquáticas, atmosféricas e antropogênicas, no caso de áreas urbanizadas - que apresentam grande variabilidade espacial e temporal nas suas contribuições relativas..$^{1-3}$ De acordo com sua origem, a matéria orgânica apresenta reatividades bastante distintas frente aos gradientes significativos nas propriedades físicoquímicas, químicas e bióticas dos sistemas aquáticos, particularmente em regiões estuarinas. ${ }^{4,5}$ A elucidação dos processos que determinam a produção, transporte e acúmulo de matéria orgânica em sistemas aquáticos é fundamental para a compreensão dos ciclos biogeoquímicos globais, mas ao mesmo tempo representa um dos grandes desafios da geoquímica orgânica em decorrência da multiplicidade dos fatores envolvidos. ${ }^{4,6}$

Diversas classes de compostos na fração de lipídios (n-alcanos, ácidos graxos, alcoóis lineares e esteróis, entre outras) são utilizadas na caracterização da matéria orgânica sedimentar devido à boa especificidade em relação à fonte e à maior resistência aos processos de degradação bacteriana, quando comparados com outros grupos de compostos orgânicos, como carboidratos e proteínas. ${ }^{3,7,8}$ Os n-alcanos, por exemplo, apresentam diferenças em relação ao tamanho da cadeia de carbono que permitem a identificação de fontes autóctonas e alóctonas de matéria orgânica, incluindo a contaminação petrogênica. ${ }^{9-11}$ Já os esterois podem ser usados para detalhar as fontes autóctonas e alóctonas naturais de matéria orgânica, ${ }^{2,12,13}$ além de serem bons indicadores do aporte de esgotos domésticos para ambientes aquáticos..$^{14,15}$

A Baía de Sepetiba $\left(23^{\circ} \mathrm{S}\right.$ e $44^{\circ} \mathrm{W}$; Figura $1 \mathrm{~S}$ - Material Suplementar) é um corpo d'água semifechado com área de $305 \mathrm{~km}^{2}$, localizado a cerca de $60 \mathrm{~km}$ ao sul da cidade do Rio de Janeiro. A bacia hidrográfica da Baía de Sepetiba ocupa uma área de 2000 km² (4,4\% da área do Estado do Rio de Janeiro) e abrange uma área montanhosa ao norte e uma extensa planície litorânea ao sul, sendo responsável

*e-mail: carreira@uerj.br pelo lançamento entre 12,6 e 14,5 milhões de $\mathrm{m}^{3} \mathrm{dia}^{-1}$ de água doce na baía, principalmente através do canal de São Francisco e rios da Guarda, Guandu e Mazomba-Cação. ${ }^{16}$ A população na região (cerca de 1.400.000 habitantes) é essencialmente urbana, em decorrência do abandono das atividades agrícolas tradicionais e substituição por atividades industriais (siderurgia e mineração) e empreendimentos imobiliários. Nos últimos 5-10 anos há uma aceleração desse processo, com a construção e/ou expansão de siderurgias e de terminais portuários voltados para exportação, assim como a ampliação do porto de Sepetiba. O turismo é outro vetor de alteração antrópica na região, particularmente na porção leste da Baía de Sepetiba. ${ }^{16}$

A contaminação de água, sedimento e biota tem sido largamente estudada na Baía de Sepetiba em relação a metais, ${ }^{17}$ mas os estudos sobre a contaminação orgânica são incipientes. ${ }^{18,19}$ Da mesma forma, as informações sobre as fontes de matéria orgânica de origem natural são relativamente restritas. ${ }^{20}$

No presente trabalho foi feita a determinação de hidrocarbonetos alifáticos e esterois em sedimentos superficiais com o objetivo de identificar as fontes naturais (autóctonas e alóctonas) e antropogênicas (hidrocarbonetos e esgotos domésticos) de matéria orgânica para a Baía de Sepetiba. Os resultados refletem a situação ambiental do início da década de 2000 , de forma que podem ser considerados como referência para futuras avaliações da contaminação orgânica nos sedimentos da Baía de Sepetiba.

\section{PARTE EXPERIMENTAL}

\section{Amostragem e preparo das amostras}

A amostragem foi realizada com busca-fundo de aço inox em setembro/2001 em 14 estações (Figura 1S - Material Suplementar), distribuídas espacialmente de forma a representar as potenciais influências de fontes naturais e antrópicas de matéria orgânica para a Baía de Sepetiba. A camada superficial $(0-2 \mathrm{~cm})$ do sedimento foi cuidadosamente sub-amostrada com espátula metálica diretamente em recipientes metálicos previamente descontaminados $\left(450{ }^{\circ} \mathrm{C}\right.$ por 
$8 \mathrm{~h}$ ), que foram mantidos sob refrigeração no campo e posteriormente estocados a $-20{ }^{\circ} \mathrm{C}$ no laboratório. As amostras foram secas em estufa $\left(60{ }^{\circ} \mathrm{C}\right)$, peneiradas em uma peneira de trama $1,25 \mathrm{~mm}$ e convertidas em pó fino $(<63 \mu \mathrm{m})$ em grau e pistilo de ágata.

\section{Determinação de carbono orgânico}

A determinação de carbono orgânico $\left(\mathrm{C}_{\text {org }}\right)$ foi realizada em analisador elementar (Carlo Erba EA 1110), seguindo método de Hedges e Stern..$^{21}$ As amostras foram previamente descarbonatadas com $\mathrm{HCl}$ $0,1 \mathrm{~mol} \mathrm{~L}^{-1}$, secas em estufa $\left(60^{\circ} \mathrm{C}\right)$ e cerca de $10 \mathrm{mg}$ (precisão $\pm 0,01$ $\mathrm{mg}$ ) pesadas diretamente em cápsulas de estanho. A quantificação foi baseada em fator de resposta do aparelho em relação a um padrão de cistina (composição de 29,99\% de C, 5,03\% de H, 11,66\% de N e $26,69 \%$ de $\mathrm{S}$ ). A precisão do método, com base na determinação de ao menos 4 réplicas de amostras selecionadas, foi de $\pm 1,7 \%$ e o limite de detecção de $0,60 \mathrm{mg} \mathrm{g}^{-1}$.

\section{Determinação de hidrocarbonetos alifáticos e esterois}

A determinação de hidrocarbonetos e esterois seguiu metodologia descrita em detalhes na literatura. ${ }^{22,23}$ Entre 5 e $10 \mathrm{~g}$ de sedimento seco foram pesados com precisão de $\pm 0,01 \mathrm{~g}$ em cápsulas de celulose e em cada amostra adicionados $50 \mu \mathrm{L}$ de solução contendo $100 \mathrm{ng} \mu \mathrm{L}^{-1}$ de cada um dos padrões sub-rogados hexadecano deuterado $\left(n-C_{16} d\right)$ e triacontano deuterado $\left(n-\mathrm{C}_{30} \mathrm{~d}\right)$ para os hidrocarbonetos alifáticos e androstanol ( $5 \alpha$-androstan- $3 \beta$-ol) para os esterois.

A extração dos hidrocarbonetos e esterois foi feita em aparelho Soxhlet durante $20 \mathrm{~h}$, usando $200 \mathrm{~mL}$ de solução 2:1 (v/v) de diclorometano:metanol (grau pesticida). O extrato bruto foi lavado com solução saturada de $\mathrm{NaCl}$, sendo os compostos de interesse reextraídos 3 vezes com $n$-hexano. $\mathrm{O}$ volume do extrato combinado foi reduzido em evaporador rotatório, sendo então adicionados cobre metálico e sulfato de cobre ativado para remoção de enxofre e traços de água, respectivamente. A separação das classes de compostos (hidrocarbonetos e esterois) foi feita em coluna de vidro preenchida com 2,5 g de sílica e 4,65 g de alumina (desativadas a 5\% com água tipo 1) e utilizando a seguinte sequência de solventes: $12 \mathrm{~mL}$ de n-hexano para a fração de hidrocarbonetos alifáticos (F1), 24 $\mathrm{mL}$ de $\mathrm{n}$-hexano:diclorometano $(1: 1, \mathrm{v} / \mathrm{v})$ para a fração de hidrocarbonetos aromáticos (não utilizada neste trabalho) e $24 \mathrm{~mL}$ de diclorometano:metanol (1:1, v/v) para a fração de esteróis (F3).

A determinação quali-quantitativa de hidrocarbonetos alifáticos e esterois foi realizada por cromatografia em fase gasosa acoplada a detector de ionização por chama (CG/DIC, HP modelo 6890). Na fração F1 (hidrocarbonetos alifáticos), avolumada a $1 \mathrm{~mL}$, foram adicionados 5000 ng de di-hidroantraceno como padrão interno de quantificação e usadas as seguintes condições analíticas no CG/DIC: coluna capilar tipo HP-5 com $30 \mathrm{~m}$ de comprimento, 0,32 $\mathrm{mm}$ de diâmetro interno e $0,25 \mu \mathrm{m}$ de espessura do filme interno; He como gás de arraste a fluxo constante de $2 \mathrm{~mL} \mathrm{~min}{ }^{-1}$; injetor a $280{ }^{\circ} \mathrm{C}$ e detector a $300{ }^{\circ} \mathrm{C}$; temperatura inicial do forno a $60^{\circ} \mathrm{C}(1 \mathrm{~min})$ e $6{ }^{\circ} \mathrm{C} / \mathrm{min}$ até $300{ }^{\circ} \mathrm{C}$, mantendo-se nessa isoterma por $10 \mathrm{~min}$. Foram identificados n-alcanos individuais na $\mathrm{F} 1$ com base nos tempos de retenção obtidos pela injeção de mistura de padrões autênticos entre $n-C_{14}$ e $n-C_{34}$ e a presença de mistura complexa não-resolvida (MCNR), caracterizada pela elevação da linha base do cromatograma (Figura 1). A quantificação de n-alcanos individuais, da MCNR e dos padrões sub-rogados foi feita por fator de resposta relativa ao padrão interno (di-idroantraceno).

A fração F3 (esterois) foi derivatizada antes da análise instrumental por reação com BSTFA (bis-trimetilsilil-trifluoracetamida) a $70^{\circ} \mathrm{C} / 1 \mathrm{~h}$. Em seguida, o extrato derivatizado foi avolumado a $1 \mathrm{~mL}$ e adicionados $5000 \mathrm{ng}$ de colestano como padrão interno. As condições instrumentais na determinação dos esterois foram iguais às utilizadas para os hidrocarbonetos alifáticos (ver acima), com exceção de um tempo maior de isoterma final (29 min). Foram identificados os seguintes esterois (Figura 2): coprostanol ( $5 \beta$-colestan-3 $\beta$-ol), colesterol (colest-5-en$3 \beta$-ol), colestanol $(5 \alpha$-colestan- $3 \beta$-ol), campesterol (24-metilcolest5 -en-3 $\beta$-ol), estigmasterol (24-etilcolest-5,22-dien-3 $\beta$-ol), sitosterol (24-etilcolest-5-en-3 $\beta$-ol) e dinosterol ( $4 \alpha, 23,24$-trimetil-colest-22-en$3 \beta$-ol). Os esterois foram identificados em relação ao tempo de retenção de padrões autênticos. Para identificar o dinosterol (não existe padrão), assim como para confirmar a identidade dos esterois e a ausência de coeluições na F3, amostras selecionadas foram também analisadas por cromatografia em fase gasosa acoplada à espectrometria de massas (CG/EM, ThermoFinnigan Polaris Trace GCQ Plus system) usando as mesmas condições analíticas da determinação de esterois por CG/ DIC. A quantificação dos esterois e do padrão sub-rogado (androstanol) foi feita por fator de resposta de padrões autênticos em relação ao padrão interno, sendo que para o dinosterol foi usado o mesmo fator de resposta do sitosterol.

Em todo o procedimento analítico foram seguidas condições de qualidade da análise, baseada na determinação de brancos de laboratório (valores encontrados foram diminuídos da análise individual), na avaliação da precisão do método ( $<20 \%$ para 4 réplicas analisadas) e no valor da recuperação do padrão sub-rogado $(70,3 \pm 15,0 \%$ para $n-C_{16} \mathrm{~d}, 85,5 \pm 9,1 \%$ para o $n-C_{30} \mathrm{de} 80,1 \pm 13,2 \%$ para o androstanol). O limite de quantificação foi calculado tendo como base a concentração do primeiro ponto da curva de calibração de cada composto e a massa média de sedimento extraída, sendo da ordem de $0,01 \mu \mathrm{g} \mathrm{g}^{-1}$ para compostos individuais (hidrocarbonetos e esterois).

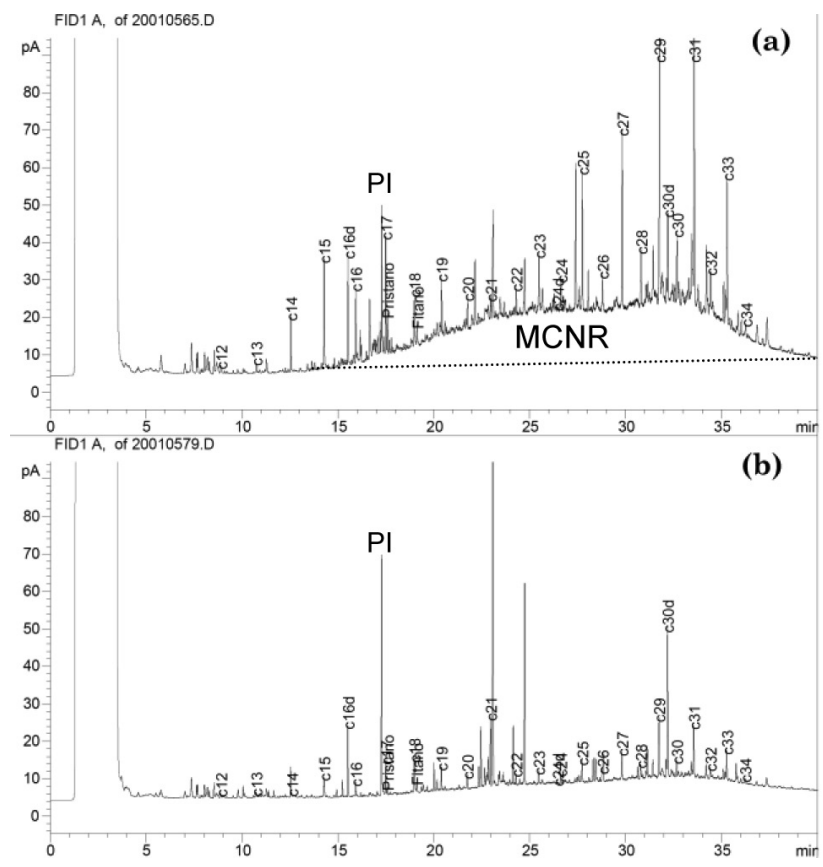

Figura 1. Exemplos de cromatogramas (CG/DIC) da fração de hidrocarbonetos alifáticos nos sedimentos das estações 123 (a) e 108 (b). A linha tracejada em (a) indica a presença de mistura complexa não-resolvida $(M C N R)$. PI = padrão interno (di-hidroantraceno)

\section{RESULTADOS}

\section{Carbono orgânico}

A concentração média de carbono orgânico $\left(\mathrm{C}_{\mathrm{org}}\right)$ para as 14 estações de coleta foi de $18,4 \pm 4,7 \mathrm{mg} \mathrm{g}^{-1}$, com variaçã̃o entre 6,6 a 


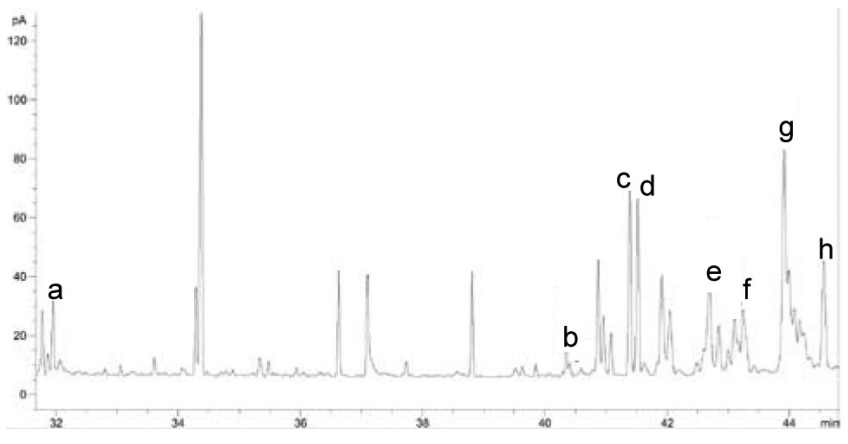

Figura 2. Exemplo de cromatograma parcial (CG/DIC) da fração de esterois para a amostra 123, indicando os seguintes compostos quantificados: androstanol (a), coprostanol (b), colesterol (c), colestanol (d), campesterol $(e)$, estigmasterol $(f)$, sitosterol $(g)$ e dinosterol $(h)$

$26,2 \mathrm{mg} \mathrm{g}^{-1}$ (Tabela 1). Apenas na estação 116 a concentração de $\mathrm{C}_{\mathrm{org}}$ é significativamente menor, o que é explicado pela sua granulometria arenosa, enquanto nas demais estações há predomínio de lama e, no caso das estações 123 e 128, lama com areia (Hélio Vilhena, comunicação pessoal). As maiores concentrações de $\mathrm{C}_{\text {org }}$ foram encontradas, de forma geral, na porção norte da área amostrada na baía (estações 119 a 133), que é a mais urbanizada e próxima ao manguezal de Coroa Grande (Figura 1S - Material Suplementar). Na porção leste da baía também há extensas áreas de manguezais (região de Guaratiba), mas as concentrações de $\mathrm{C}_{\text {org }}$ no sedimento são menores $\left(13,6 \mathrm{mg} \mathrm{g}^{-1}\right.$ na estação 102; Tabela 1) em comparação com a porção oeste da baía.

\section{Hidrocarbonetos alifáticos}

Os hidrocarbonetos alifáticos foram caracterizados em relação à distribuição de n-alcanos individuais e à presença de mistura complexa não-resolvida (MCNR). Outros compostos individuais na fração de hidrocarbonetos alifáticos foram observados nos cromatogramas (Figura 1), mas não foram quantificados ou não serão considerados no presente trabalho.
Os n-alcanos ocorreram na faixa entre $n-C_{14}$ e $n-C_{34}$, com concentrações totais entre 0,26 e $2,65 \mu \mathrm{g} \mathrm{g}^{-1}$ (Tabela 1). As estações 119, 123, 128 e 133, localizadas na porção norte da baía (Figura 1S, Material Suplementar), apresentaram os valores mais altos para o somatório de n-alcanos. Essas mesmas estações também possuem os maiores teores de carbono orgânico, mas não foi observada correlação significativa entre carbono e total de n-alcanos quando é considerado o conjunto de amostras analisadas $(\mathrm{r}=0,52$; $\mathrm{p}=0,06 ; \mathrm{N}=14)$.

A distribuição dos n-alcanos individuais é do tipo bimodal, com predomínio dos homólogos ímpares na faixa $n-C_{25}$ a n- $C_{33}$ e, em segundo lugar, entre $n-C_{14}$ e $n-C_{18}$. Em todas as amostras foi encontrado predomínio dos n-alcanos $n-\mathrm{C}_{29}$ ou n- $\mathrm{C}_{31}$ (Figura 3). O n- $\mathrm{C}_{21}$ não foi quantificado devido à coeluição com outro composto, possivelmente um alceno isoprenoide multirramificado $\left(\mathrm{HBI} \mathrm{C} \mathrm{C}_{25} \mathrm{H}_{46}\right)$, como observado na mesma região por Figueiredo e colaboradores. ${ }^{19}$

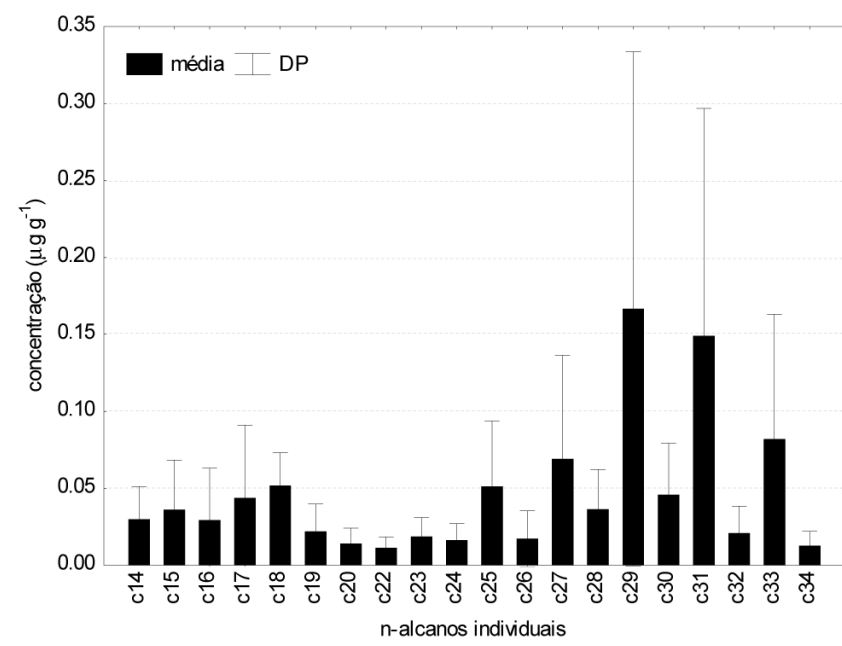

Figura 3. Concentrações médias ( \pm desvio-padrão) de n-alcanos individuais nos sedimentos da Baía de Sepetiba $(N=14)$

Tabela 1. Concentração de carbono orgânico $\left(\mathrm{mg} \mathrm{g}^{-1}\right)$, hidrocarbonetos alifáticos $\left(\sum \mathrm{n}\right.$-alcanos $\left.{ }^{\mathrm{a}} \mathrm{e}_{\mathrm{MCNR}} \mu_{\left.\mathrm{g} \mathrm{g}^{-1}\right)}\right)$ e esterois individuais $\left(\mu \mathrm{gg} \mathrm{g}^{-1}\right)$

\begin{tabular}{|c|c|c|c|c|c|c|c|c|c|c|c|c|c|c|}
\hline Amostra & 102 & 103 & 105 & 106 & 108 & 109 & 111 & 112 & 115 & 116 & 119 & 123 & 128 & 133 \\
\hline $\begin{array}{l}\text { Carbono } \\
\text { orgânico }\end{array}$ & 13,6 & 18,2 & 14,9 & 16,6 & 22,8 & 20,5 & 17,4 & 19,2 & 20,3 & 6,6 & 23,5 & 19,9 & 17,9 & 26,2 \\
\hline \multicolumn{15}{|l|}{$\begin{array}{l}\text { Hidroc. } \\
\text { Alifáticos }\end{array}$} \\
\hline$\sum$ n-alcanos & 0,63 & 0,52 & 0,62 & 0,31 & 0,61 & 0,26 & 0,29 & 0,37 & 0,82 & 0,53 & 2,65 & 1,81 & 1,47 & 2,07 \\
\hline MCNR & bnd & nd & nd & nd & 8,57 & nd & 2,08 & nd & 8,85 & nd & 13,16 & 17,41 & 12,26 & 11,34 \\
\hline \multicolumn{15}{|l|}{ Esteróis } \\
\hline coprostanol & 0,13 & 0,05 & 0,07 & 0,01 & 0,05 & 0,01 & 0,03 & 0,03 & 0,04 & 0,02 & 0,13 & 0,15 & 0,42 & 0,13 \\
\hline colesterol & 0,16 & 0,17 & 0,14 & 0,08 & 0,23 & 0,09 & 0,07 & 0,12 & 0,15 & 0,13 & 0,55 & 0,83 & 1,02 & 0,69 \\
\hline colestanol & 0,23 & 0,29 & 0,07 & 0,10 & 0,33 & 0,14 & 0,06 & 0,18 & 0,18 & 0,16 & 1,23 & 0,80 & 1,05 & 1,49 \\
\hline campesterol & 0,06 & 0,28 & 0,09 & 0,25 & 0,24 & 0,22 & 0,07 & 0,16 & 0,18 & 0,13 & 0,63 & 0,69 & 0,81 & 0,77 \\
\hline estigmasterol & 0,04 & 0,21 & 0,05 & 0,06 & 0,15 & 0,06 & 0,04 & 0,09 & 0,15 & 0,06 & 0,48 & 0,43 & 0,75 & 0,55 \\
\hline sitosterol & 0,18 & 0,58 & 0,25 & 0,28 & 0,47 & 0,32 & 0,36 & 0,36 & 0,52 & 0,46 & 3,06 & 1,72 & 4,14 & 1,68 \\
\hline dinosterol & 0,25 & 0,91 & 0,25 & 0,49 & 0,50 & 0,55 & 0,14 & 0,47 & 0,28 & 0,33 & 0,97 & 0,99 & 1,05 & 1,69 \\
\hline total esteróis & 1,05 & 2,48 & 0,92 & 1,27 & 1,98 & 1,40 & 0,77 & 1,40 & 1,51 & 1,28 & 7,06 & 5,62 & 9,24 & 7,00 \\
\hline
\end{tabular}

${ }^{\mathrm{a}} \mathrm{n}$-alcanos individuais entre $\mathrm{n}-\mathrm{C}_{14}$ a n- $\mathrm{C}_{34}{ }^{\text {b }} \mathrm{nd}=$ não-detectado 
A mistura complexa não-resolvida (MCNR), caracterizada pela elevação da linha base dos cromatogramas na fração de alifáticos (Figura 1a) devido à presença de compostos ramificados e cíclicos, ${ }^{24}$ foi detectada em $50 \%$ das amostras, com concentração média de 10,52 $\pm 4,76 \mu \mathrm{g} \mathrm{g}^{-1}$ (Tabela 1). Os maiores valores foram medidos nas estações da porção norte da baía $(119,123,128$ e 133), que apresentaram as maiores concentrações de n-alcanos, e também foi detectada nas estações próximas aos principais rios da região (108, 111 e 115). A MCNR apresentou um perfil unimodal e distribuição na faixa entre n- $\mathrm{C}_{18}$ e n- $\mathrm{C}_{35}$, com máximo entre $\mathrm{n}-\mathrm{C}_{29}$ ou n- $\mathrm{C}_{31}$, repetindo resultados prévios para a mesma região. ${ }^{19}$

\section{Esterois}

A concentração do total de esterois variou de 0,77 a $9,24 \mu \mathrm{g} \mathrm{g}^{-1}$ (Tabela 1), com valores $>5,62 \mu \mathrm{g} \mathrm{g}^{-1}$ nas estações 119, 123, 128 e 133 . Nestas mesmas estações também foram encontradas as concentrações mais altas de carbono orgânico e do total de hidrocarbonetos alifáticos.

O 24-etilcolest-5-en-3 $\beta$-ol (sitosterol; $29,4 \pm 9,4 \%$ ) e o $4 \alpha, 23,24$-trimetilcolest-22-en-3 $\beta$-ol (dinosterol; 24,7 $\pm 9,0 \%$ ) foram os compostos mais abundantes, mas ambos apresentaram distribuição inversamente proporcional $(\mathrm{r}=-0,69 ; \mathrm{p}<0,01 ; \mathrm{N}=$ 14) entre as estações (Figura 4). O sitosterol foi mais abundante nas estações 111, 115, 116, 119, 123, 128 e 133, localizadas na porção norte da baía e/ou próximas aos principais rios da região e que têm, na maioria dos casos, as maiores concentrações de esterois totais. Já o dinosterol foi mais abundante em estações com menores concentrações de esterois totais, como as 106, 109 e 112, mais afastadas da linha de costa, ou nas estações 102 e 103, na porção leste da baía (Figura 4).

O colest-5-en-3 $\beta$-ol (colesterol), normalmente o mais abundante em ambientes aquáticos por ser o principal esterol no plâncton, particularmente crustáceos, ${ }^{12}$ representou apenas $9,9 \pm 2,8 \%$ do total de esterois. Possivelmente, o baixo percentual de colesterol deve-se à transformação em colestanol ( $5 \alpha$-coletan- $3 \beta$-ol), que representou $12,9 \pm 4,6 \%$ do total de esterois. O $5 \beta$-colestan-3 $\beta$-ol (coprostanol), derivado de esgotos domésticos, ${ }^{25,26}$ representou apenas $3,2 \pm 3,0 \%$ do total de esterois quantificados no sedimento da Baía de Sepetiba.

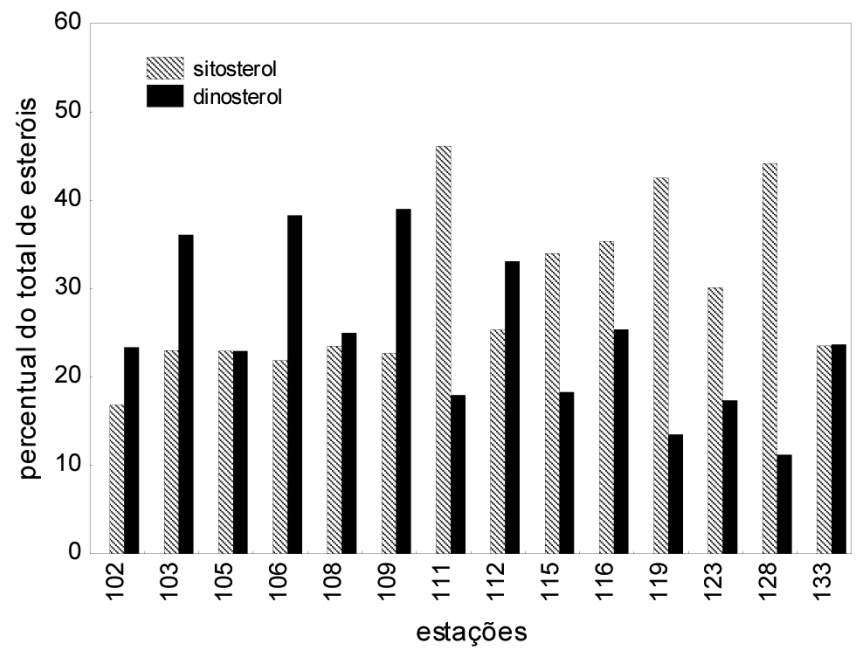

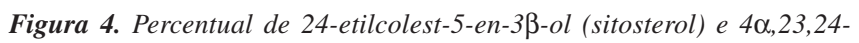
trimetilcolest-22-en-3ß-ol (dinosterol) para o total de esterois quantificados

\section{DISCUSSÃO}

Os sedimentos da Baía de Sepetiba apresentam concentrações de carbono orgânico entre 6,6 e $26,2 \mathrm{mg} \mathrm{g}^{-1}$ (Tabela 1), inferiores, portanto, às de sedimentos lamosos de outras áreas costeiras com elevado impacto antrópico, como a Baía de Guanabara, no Rio de Janeiro (20-60 mg g $\left.{ }^{-1}\right)^{22,27}$ ou o estuário do Rio Capiberibe, em Pernambuco $\left(6,0-39 \mathrm{mg} \mathrm{g}^{-1}\right) .{ }^{28} \mathrm{O}$ relativo empobrecimento de carbono orgânico nos sedimentos da Baía de Sepetiba, apesar da alta carga orgânica de natureza antrópica, particularmente pelos rios da parte central (canal São Francisco e rio Guandu), possivelmente está associado às condições hidrodinâmicas locais, que favorecem a dispersão dos contaminantes, ${ }^{18}$ mas também reflete condições ambientais menos severas do que nas outras áreas citadas. Apesar das baixas concentrações, os marcadores utilizados no presente trabalho (hidrocarbonetos alifáticos e esterois) permitiram identificar as fontes naturais e antrópicas de matéria orgânica para a Baía de Sepetiba, como será detalhado a seguir.

\section{Contribuição de matéria orgânica autóctona e alóctona natural}

A presença de cadeias longas de n-alcanos e com número ímpar de carbono nas ceras epicuticulares das folhas de plantas superiores ${ }^{11,29}$ sugere o uso destes compostos como indicadores da origem da matéria orgânica. Esse padrão de composição de n-alcanos é encontrado em sedimentos marinhos em locais onde o aporte terrígeno de matéria orgânica é significativo, enquanto o aporte de matéria orgânica de origem planctônica é caracterizado pela presença de n-alcanos leves, principalmente em n- $\mathrm{C}_{17}{ }^{9,30}$

As concentrações de n-alcanos totais na Baía de Sepetiba $(0,26$ e 2,65 $\mu \mathrm{g} \mathrm{g}^{-1}$ ) são da mesma ordem de grandeza das encontradas em outras áreas costeiras no Brasil, como na Lagoa dos Patos $(0,20$ a $\left.7,50 \mu \mathrm{g} \mathrm{g}^{-1}\right)^{31}$, baía e estuário de Santos $\left(0,10 \text { a 14,56 } \mu \mathrm{g} \mathrm{g}^{-1}\right)^{32}$ e no canal de São Sebastião, nas proximidades de terminal de petróleo $\left(0,04\right.$ a $\left.8,53 \mu \mathrm{g} \mathrm{g}^{-1}\right)$, mas são significativamente inferiores às de áreas contaminadas por hidrocarbonetos, como na porção interna do estuário de Santos $\left(3,91 \text { a } 114 \mu \mathrm{g} \mathrm{g}^{-1}\right)^{33}$ ou na Baía de Guanabara $\left(1,24\right.$ a $\left.56,7 \mu \mathrm{g} \mathrm{g}^{-1}\right) .{ }^{34}$ Os valores também são inferiores aos encontrados em sedimentos com aporte significativo de matéria orgânica derivada de vegetais superiores (manguezais), como no estuário de Cananéia, que apresenta concentrações de n-alcanos totais entre 4,4 e $158 \mu \mathrm{g} \mathrm{g}^{-1} .^{35}$

É interessante notar que as concentrações de n-alcanos totais nas estações 111 e $115\left(0,29-0,82 \mu \mathrm{g} \mathrm{g}^{-1}\right.$; Tabela 1$)$, localizadas nas proximidades dos principais rios contribuintes à baía, são uma ordem de grandeza inferior à faixa de 5,92 a 8,56 $\mu \mathrm{g} \mathrm{g}^{-1}$ encontrada em estações mais próximas à desembocadura desses rios. ${ }^{19}$ Este gradiente significativo de concentração possivelmente é decorrente da rápida remoção dos n-alcanos para os sedimentos durante o processo de mistura estuarina ou reflete a dispersão eficiente dos materiais lançados pelos rios da porção central da baía. ${ }^{18}$

As maiores concentrações de n-alcanos de cadeia ímpar de carbono na faixa $n-C_{25}$ e $n-C_{33}$ (Figura 3) e os valores do índice preferencial de carbono ${ }^{36}$ entre 3,0 e 4,5 (Tabela 2) indicam que o aporte de matéria orgânica de origem continental é mais significativo que o aporte autóctono. ${ }^{37} \mathrm{O}$ índice ACL (tamanho médio da cadeia, na sigla em inglês), que correlaciona a proporção entre os n-alcanos de maior peso molecular, pode ser usado para caracterizar o aporte de matéria orgânica alóctona. ${ }^{38} \mathrm{O}$ valor médio de ACL calculado para os sedimentos da Baía de Sepetiba foi de 30,0 \pm 0,13 (Tabela 2), o que se situa na faixa encontrada em folhas de duas espécies de manguezais da região: A. schauerianna com 30,6 0 0,4 e R. mangle, com 29,5 $\pm 0,2,{ }^{39}$ e é inferior ao encontrado no petróleo $(\mathrm{ACL}=$ 28,0). ${ }^{10}$ Assim, o índice ACL indica que os manguezais presentes no entorno da baía são a principal fonte de matéria orgânica alóctona para a Baía de Sepetiba, possivelmente como uma mistura de $A$. schauerianna e R. mangle. 
Tabela 2. Índices diagnósticos entre os hidrocarbonetos alifáticos e esterois nos sedimentos superficiais da Baía de Sepetiba

\begin{tabular}{|c|c|c|c|c|c|c|c|c|c|c|c|c|c|c|}
\hline Estações & 102 & 103 & 105 & 106 & 108 & 109 & 111 & 112 & 115 & 116 & 119 & 123 & 128 & 133 \\
\hline \multicolumn{15}{|l|}{ Hidr. Alifáticos ${ }^{a}$} \\
\hline $\operatorname{IPC}\left(>n-C_{24}\right)$ & 0,66 & 2,27 & 2,78 & 2,98 & 3,07 & 2,55 & 3,68 & 3,31 & 3,40 & 4,19 & 4,47 & 4,29 & 4,12 & 4,18 \\
\hline ACL & 29,9 & 30,1 & 30,1 & 30,0 & 30,0 & 30,1 & 29,9 & 30,2 & 29,8 & 30,0 & 30,0 & 29,9 & 30,1 & 30,3 \\
\hline TAR & 1,0 & 4,7 & 5,6 & 1,3 & 2,2 & 1,7 & 8,0 & 2,8 & 3,7 & 8,8 & 4,3 & 4,8 & 7,3 & 2,6 \\
\hline $\mathrm{MCNR} / \Sigma \mathrm{n}$-alc & - & - & - & - & 14,15 & - & 7,09 & - & 10,82 & - & 4,97 & 9,62 & 8,36 & 5,49 \\
\hline \multicolumn{15}{|l|}{ Esterois $^{b}$} \\
\hline cop/colesterol & 0,81 & 0,29 & 0,50 & 0,12 & 0,20 & 0,15 & 0,51 & 0,21 & 0,29 & 0,17 & 0,24 & 0,17 & 0,42 & 0,19 \\
\hline $5 \beta /(5 \beta+5 \alpha)$ estanol & 0,36 & 0,14 & 0,48 & 0,08 & 0,12 & 0,09 & 0,35 & 0,13 & 0,19 & 0,13 & 0,10 & 0,15 & 0,29 & 0,08 \\
\hline
\end{tabular}

${ }^{a} \mathrm{MCNR} / \sum \mathrm{n}$-alc: $\mathrm{MCNR} / \mathrm{soma}$ de n-alcanos na faixa $\mathrm{n}-\mathrm{C}_{14}$ a n- $\mathrm{C}_{34} ; \mathrm{IPC}\left(>\mathrm{n}-\mathrm{C}_{24}\right)$ : índice preferencial de carbono com n-alcanos acima de $\mathrm{n}-\mathrm{C}_{24}$; TAR (razão terrestre/aquático): $\left(\mathrm{n}-\mathrm{C}_{27}+\mathrm{n}-\mathrm{C}_{29}+\mathrm{n}-\mathrm{C}_{31}\right) /\left(\mathrm{n}-\mathrm{C}_{15}+\mathrm{n}-\mathrm{C}_{17}+\mathrm{n}-\mathrm{C}_{19}\right) ; \mathrm{ACL}$ (comprimento médio da cadeia): $\sum$ (Ci*[Ci $) / \sum$, onde

[Ci] é a concentração do n-alcano com número de carbono Ci, na faixa de 27 a 33. ${ }^{\mathrm{b}} \mathrm{cop} / \mathrm{colesterol:} \operatorname{coprostanol} / \mathrm{colesterol} ; 5 \beta /(5 \beta+5 \alpha)$ estanol: coprostanol/(coprostanol+colestanol)

A presença de n-alcanos entre $n-C_{14}$ e n- $C_{19}$ (Figura 3), embora em menores concentrações em comparação com os compostos acima de $n-\mathrm{C}_{24}$, indica o acúmulo de material planctônico, possivelmente já alterado pela atividade bacteriana, uma vez que não há predomínio de compostos ímpares ou pares entre os n-alcanos leves. ${ }^{1,40} \mathrm{O}$ índice TAR (terrestrial aquatic ratio) foi proposto originalmente por Bourboniere e Meyers ${ }^{41}$ para relacionar a proporção dos n-alcanos de origem terrestre $\left(n-C_{29}, n-C_{29}\right.$ e n- $\left.C_{31}\right)$ e marinha $\left(n-C_{15}, n-C_{17}\right.$ e $n-\mathrm{C}_{19}$ ). Na Baía de Sepetiba, os valores de TAR mais elevados, entre 4 e 9 (Tabela 2), foram encontrados nas mesmas estações nas quais os outros índices de n-alcanos já discutidos (IPC e ACL) também indicam presença de matéria orgânica terrestre. Já nas estações mais afastadas da linha de costa (106, 109 e 112) e nas estações 102, 108 e 133, o TAR é bem inferior, entre 1,0 e 2,2 (Tabela 2), em função da maior contribuição de n-alcanos leves e, consequentemente, da presença de matéria orgânica planctônica nessas estações, a despeito das indicações de alteração bacteriana dos n-alcanos e sua possível influência sobre o TAR.

A distribuição dos esterois é marcada pela ocorrência de concentrações mais altas nas estações 119 a $133\left(5,7\right.$ a 9,4 $\left.\mu \mathrm{g} \mathrm{g}^{-1}\right)$, sendo o 24-etilcolest-5-en-3 $\beta$-ol (sitosterol) o esterol mais abundante em todas elas. A origem do sitosterol em sistemas aquáticos é relativamente dúbia porque não é possível identificar, nas condições analíticas usuais, a posição isomérica do grupo 24-etil: o 24- $\alpha$ (clionasterol) é derivado do plâncton, enquanto o $24-\beta$ ( $\beta$-sitosterol) é produzido por vegetais superiores. ${ }^{7}$ A razão entre 24 -metilcolest-5-en-3 $\beta$-ol (campesterol), 24-etilcolest-5,22-dien-3 $\beta$-ol (estigmasterol) e o 24-etilcolest-5-en-3 $\beta$-ol (sitosterol) pode ser usada para identificar a origem do sitosterol: entre 1:1,4:4,0 e 1:1,6:6,0 a origem é continental, enquanto razões inferiores são atribuídas ao plâncton marinho..$^{42}$ De acordo com esses valores de referência, nas estações 111, 116, 119 e 128 o sitosterol tem origem terrestre, enquanto nas demais - incluindo as estações 123 e 133 - pode haver mistura entre fontes autóctonas e alóctonas. Além disto, houve predomínio de sitosterol nas mesmas estações com altas concentrações de n-alcanos derivado de vegetação superior. Esses resultados são evidências da importância do aporte alóctono na Baía de Sepetiba.

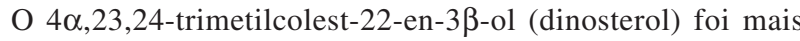
abundante que o sitosterol nas estações com menores concentrações de esterois totais $\left(0,78\right.$ a $2,50 \mu \mathrm{g} \mathrm{g}^{-1}$; Tabela 1$)$, que inclui aquelas mais afastadas da linha de costa $(106,109$ e 112) e as localizadas na porção leste da baía (102 e 103). Nas estações 108 e 133, os dois esterois foram encontrados em proporções semelhantes (Figura
4). O dinosterol é um marcador inequívoco de matéria orgânica planctônica, pois é derivado de dinoflagelados e de alguns grupos de diatomáceas. ${ }^{7,12}$ Portanto, a ocorrência de dinosterol nas estações citadas, somada à maior concentração de n-alcanos leves nas mesmas estações, mostra que ao se afastar da linha de costa e na porção leste da baía há maior influência de fontes internas (produção planctônica) em relação às externas (vegetação terrestre) no acúmulo da matéria orgânica sedimentar.

\section{Contaminação por hidrocarbonetos de petróleo}

A contribuição de hidrocarbonetos de origem antrópica pode ser avaliada pela distribuição dos n-alcanos e pela presença de mistura complexa não-resolvida (MCNR). Os n-alcanos no petróleo distribuem-se de forma relativamente uniforme entre cadeias pares e ímpares, reflexo do processo natural de sua formação, enquanto a síntese biogênica produz n-alcanos ímpares. ${ }^{11,43} \mathrm{Na}$ seção anterior foi amplamente discutido que os n-alcanos nos sedimentos da Baía de Sepetiba são de origem biogênica, sem indicação clara de aporte de origem antrópica.

A ocorrência de mistura complexa não-resolvida é um indicador consistente da presença de resíduos de petróleo, normalmente já em processo de intemperização. ${ }^{44}$ No presente trabalho, a MCNR foi encontrada nas estações que apresentaram as maiores concentrações de carbono orgânico, n-alcanos e esterois e que são localizadas próximas ao porto de Sepetiba (119 e 123) e de áreas mais densamente urbanizadas e com movimentação significativa de pequenas embarcações de pesca e de turismo (Figura 2S, Material Suplementar). Em todas essas estações, a razão MCNR/ $\sum$ n-alcanos variou entre 4,97 e 14,1 (Tabela 2). Embora esses valores sejam superestimados em relação ao índice originalmente proposto (MCNR/alifáticos resolvidos totais), ${ }^{37,45}$ elas apontam para origem petrogênica da MCNR devido ao seu predomínio em relação ao total de n-alcanos.

A despeito da indicação de aportes petrogênicos em determinadas estações (Figura 2S, Material Suplementar), a faixa de concentração de MCNR na Baía de Sepetiba (2,08 a 17,41 $\left.\mu \mathrm{g} \mathrm{g}^{-1}\right)$ é de uma a duas ordens de grandeza inferior à observada em áreas com contaminação petrogênica mais elevada, como a Baía de Guanabara (38 a $2.127 \mu \mathrm{g}$ $\left.\mathrm{g}^{-1}\right)^{34}$ ou o estuário interno da Baía de Santos $\left(39,7\right.$ a $\left.2.015 \mu \mathrm{g} \mathrm{g}^{-1}\right){ }^{33}$ Figueiredo e colaboradores ${ }^{19}$ reportam concentrações de MCNR entre 36,7 e $179 \mu \mathrm{g} \mathrm{g}^{-1}$ para estações na Baía de Sepetiba próximas ao rio da Guarda e canais de São Francisco e do Guandu. Essas concentrações diminuem para 2,0 a $8,85 \mu \mathrm{g} \mathrm{g}^{-1}$ nas estações a 2-3 
$\mathrm{km}$ dos rios $(108,111$ e 115$)$ e para não-detectado para estações a 5-7 km de distância (109, 112 e 116). Portanto, há um gradiente de concentração da MCNR no sentido perpendicular à costa e em relação à desembocadura dos rios, sendo bem mais acentuado para a MCNR do que o descrito para os n-alcanos.

\section{Aporte de esgotos domésticos}

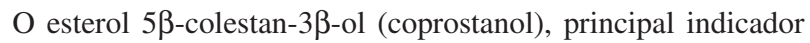
da contaminação fecal, ${ }^{14,23}$ foi detectado em todas as amostras de sedimento coletadas na Baía de Sepetiba. As maiores concentrações $\left(0,13\right.$ a $0,42 \mu \mathrm{g} \mathrm{g}^{-1}$; Tabela 1$)$ foram encontradas nas estações $119 \mathrm{a}$ 133, as mesmas com contaminação petrogênica. Essas estações são as mais próximas dos principais centros urbanos no entorno da Baía de Sepetiba, com destaque para a estação 128, em frente ao município de Itacuruçá (Figura 3S, Material Suplementar).

De forma geral, os resultados de coprostanol para a Baía de Sepetiba indicam ausência ou baixo nível de contaminação, uma vez que apenas valores acima de $0,50 \mu \mathrm{g} \mathrm{g}^{-1}$ são apontados como indicativos de acúmulo de material de origem fecal no sedimento. ${ }^{46}$ Além disto, em todas as estações as razões coprostanol/colesterol e $5 \beta /(5 \beta+5 \alpha)$ estanol (coprostanol/coprostanol + colestanol) foram inferiores a 1,0 e 0,7 (Tabela 3), o que confirma que a contaminação fecal na Baía de Sepetiba ainda é incipiente. ${ }^{26}$

Em comparação com outras áreas costeiras no Brasil (Tabela 3), as concentrações de coprostanol na Baía de Sepetiba são bastante inferiores, por exemplo, às da Baía de Guanabara $(0,33$ a $105 \mu \mathrm{g}$ $\left.\mathrm{g}^{-1}\right),{ }^{23,47-49}$ que é a região com o maior nível de contaminação fecal no país, mas são próximas a regiões como a Lagoa dos $\operatorname{Patos}^{50}$ e a enseada de Santos ${ }^{51,52}$.

Tabela 3. Coprostanol em sedimentos de diferentes regiões na costa brasileira

\begin{tabular}{lccc}
\hline Local & $\begin{array}{c}\text { Camada sedi- } \\
\text { mento }(\mathrm{cm})\end{array}$ & $\begin{array}{c}\text { Faixa de concentração } \\
\left(\mu \mathrm{g} \mathrm{g}^{-1}\right)\end{array}$ & Ref. \\
\hline $\begin{array}{l}\text { Lagoa dos Patos } \\
\text { (RS) }\end{array}$ & $0-2$ & $<0,001^{\mathrm{a}}-0,92$ & 50 \\
$\begin{array}{l}\text { Florianópolis } \\
\text { (SC) }\end{array}$ & $0-5$ & nd $^{\mathrm{b}}-2,88$ & 53 \\
$\begin{array}{l}\text { Litoral Norte do } \\
\text { Estado de São }\end{array}$ & $0-15$ & $0,02-5,90$ & 54 \\
$\begin{array}{l}\text { Paulo } \\
\text { Ubatuba (SP) }\end{array}$ & $0-3$ & $<0,01 *-0,27$ & 55 \\
$\begin{array}{l}\text { Enseada de San- } \\
\text { tos e região }\end{array}$ & $0-2$ & $<0,01-1,55$ & 51 \\
$\begin{array}{l}\text { Estuário } \\
\text { Capibaribe }\end{array}$ & $0-3$ & $0,52-7,30$ & 28 \\
$\begin{array}{l}\text { Recife/PE) } \\
\begin{array}{l}\text { Norte da Baía } \\
\text { de Guanabara }\end{array}\end{array}$ & $0-3$ & $0,33-40,0$ & 23 \\
$\begin{array}{l}\text { Sudoeste da } \\
\text { Baía de }\end{array}$ & $0-2$ & $1,38-105$ & 49 \\
$\begin{array}{l}\text { Guanabara } \\
\text { Sudeste da Baía } \\
\text { de Guanabara }\end{array}$ & $0-5$ & $0,64-36,2$ & 47 \\
$\begin{array}{l}\text { Baía de } \\
\text { Sepetiba (RJ) }\end{array}$ & $0-2$ & $0,01-0,42$ & presente \\
\hline
\end{tabular}

${ }^{\mathrm{a}}$ limite de quantificação; ${ }^{\mathrm{b}}$ nd = não detectado

\section{CONCLUSÕES}

A determinação de hidrocarbonetos alifáticos e esteróis permitiu avaliar as contribuições de matéria orgânica autóctona e alóctona para os sedimentos da Baía de Sepetiba, incluindo a influência de atividades antrópicas. Foi observado que os aportes de origem continental, principalmente de florestas de manguezais, são o principal contribuinte de matéria orgânica para os sedimentos. $\mathrm{O}$ aporte de matéria orgânica planctônica, embora menos significativo, também foi identificado, particularmente nas estações mais distantes da linha de costa.

A presença de hidrocarbonetos de petróleo e de esgotos domésticos foi identificada nas regiões próximas ao porto de Sepetiba e nas áreas mais urbanizadas, mas o nível de contaminação referente ao início da década de 2000 era reduzido em comparação com outras áreas costeiras no país.

Recomenda-se, em trabalhos futuros, a consideração de outros esterois derivados da produção planctônica para melhor avaliação do aporte de matéria autóctona para a Baía de Sepetiba, assim como a coleta de sedimentos depositados nos últimos 5 anos para avaliar a evolução do quadro de contaminação orgânica nos sedimentos da Baía de Sepetiba.

\section{MATERIAL SUPLEMENTAR}

Como material suplementar, disponível gratuitamente em http:// quimicanova.sbq.org.br, na forma de arquivo pdf, são apresentados os mapas da área estudada (Figura 1S) e da distribuição espacial das concentrações de MCNR (Figura 2S) e de coprostanol (Figura 3S).

\section{AGRADECIMENTOS}

À Fundação Carlos Chagas Freitas de Amparo à Pesquisa do Estado do Rio de Janeiro (FAPERJ) pelo apoio financeiro a R. Carreira, através do programa Prociência. Os comentários de 2 revisores anônimos contribuíram para melhorar a versão final do manuscrito.

\section{REFERÊNCIAS}

1. Xu, Y.; Mead, R.; Jaffé, R.; Hydrobiologia 2006, 569, 179.

2. Waterson, E. J.; Canuel, E. A.; Org. Geochem. 2008, 39, 422.

3. Volkman, J. K.; Revill, A. T.; Bonham, P. I.; Clementson, L. A.; Org. Geochem. 2007, 38, 1039.

4. Bianchi, T. S.; Canuel, E. A.; Org. Geochem. 2001, 32, 451.

5. Hobbie, J. E., ed.; Estuarine Science - A Synthetic Approach to Research and Practice, Island Press: Covelo, 2000; Goñi, M. A.;Teixeira, M. J.;Perkey, D. W.; Estuar. Coast. Shelf Sci. 2003, 57, 1023.

6. Hedges, J. I.; Keil, R. G.; Mar. Chem. 1999, 65, 55; Hedges, J. I.; Keil, R. G.; Benner, R.; Org. Geochem. 1997, 27, 195.

7. Volkman, J. K. Em Handbook of Environmental Chemistry, Volume 2: Reactions and Processes; Hutzinger, O.; Kostianoy, A.; Barceló, D., eds.; Springer-Verlag, 2006, p.27-70

8. Meyers, P. A.; Org. Geochem. 2003, 34, 261; Saliot, A.; Laureillard, J.; Scribe, P.; Sicre, M. A.; Mar. Chem. 1991, 36, 233; Countway, R. E.; Canuel, E. A.; Dickhut, R. M.; Org. Geochem. 2007, 38, 365; McCallister, S. L.; Bauer, J. E.; Ducklow, H. W.; Canuel, E. A.; Org. Geochem. 2006, 37, 454.

9. Gao, X.; Chen, S.; Xie, X.; Long, A.; Ma, F.; Environ. Pollut. 2007, 148, 40.

10. Jeng, W.-L.; Mar. Chem. 2006, 102, 242.

11. Reddy, C. M.; Eglinton, T. I.; Palic, R.; Benitez-Nelson, B. C.; Stojanovic, G.; Palic, I.; Djordjevic, S.; Eglinton, G.; Org. Geochem. 2000, 31, 331 . 
12. Volkman, J. K.; Barret, S. M.; Blackburn, S. I.; Mansour, M. P.; Sikes, E. L.; Gelin, F.; Org. Geochem. 1998, 29, 1163.

13. Zimmerman, A. R.; Canuel, E. A.; Limnol. Oceanog. 2002, 47, 1084.

14. Shah, V. G.; Dunstan, R. H.; Geary, P. M.; Coombes, P.; Roberts, T. K.; Von Nagy-Felsobuki, E.; Water Res. 2007, 41, 3691; Readman, J. W.; Fillmann, G.; Tolosa, I.; Bartocci, J.; Mee, L. D.; Mar. Pollut. Bull. 2005, 50,310 .

15. Isobe, K. O.; Tarao, M.; Chiem, N. H.; Min, L. Y.; Takada, H.; Appl. Environ. Microbiol. 2004, 70, 814.

16. SEMA, Secretaria de Estado de Meio Ambiente do Rio de Janeiro; Relatório Final - Parte I: diagnóstico ambiental, 1998.

17. Lacerda, L. D.; Marins, R. V.; Paraquetti, H. H. M.; Mounier, S.; Benaim, J.; Fevrier, D.; J. Braz. Chem. Soc. 2001, 12, 93; Lacerda, L. D.; Molisani, M. M.; Mar. Pollut. Bull. 2006, 52, 974; Paraquetti, H. H. M.; Lacerda, L. D.; Almeida, M. D.; Marins, R. V.; Mounier, S.; J. Braz. Chem. Soc. 2007, 18, 1259.

18. Cunha, C. L. N.; Rosman, P. C. C.; Ferreira, A. P.; Monteiro, C. N. T.; Cont. Shelf Res. 2006, 26, 1940.

19. Figueiredo, L. H. M.; Wagener, A. L. R.; Dagaut, J.; Saliot, A.; J. Braz. Chem. Soc. 2008, 19, 516.

20. Rezende, C. E.; Lacerda, L. D.; Ovalle, A. R. C.; Silva, L. F. F.; Braz. J. Biology 2007, 67, 673; Luciani, X.; Mounier, S.; Paraquetti, H. H. M.; Redon, R.; Lucas, Y.; Bois, A.; Lacerda, L. D.; Raynaud, M.; Ripert, M.; Mar. Environ. Res. 2008, 65, 148.

21. Hedges, J. I.; Stern, J. H.; Limnol. Oceanog. 1984, 29, 657.

22. Carreira, R. S.; Wagener, A. L. R.; Fileman, T.; Readman, J.; Macko, S. A.; Veiga, A.; Mar. Chem. 2002, 79, 207.

23. Carreira, R. S.; Wagener, A. L. R.; Readman, J. W.; Estuar. Coast. Shelf Sci. 2004, 60, 587.

24. Gough, M. A.; Rowland, S. J.; Nature 1990, 344, 648; Sutton, P. A.; Lewis, C. A.; Rowland, S. J.; Org. Geochem. 2005, 36, 963.

25. Huang, W. Y.; Meinschein, W. G.; Geochim. Cosmochim. Acta 1979, 43, 739.

26. Grimalt, J. O.; Fernandez, P.; Bayona, J. M.; Albaiges, J.; Environ. Sci. Technol. 1990, 24, 357; Takada, H.; Eganhouse, R. P. Em Encyclopedia of Environmental Analysis and Remediation; Meyers, R. A., ed.; John Wiley \& Sons, Inc.:New York, 1998.

27. Neto, J.; Gingele, F.; Leipe, T.; Brehme, I.; Environ. Geol. 2006, 49, 1051.

28. Fernandes, M. B.; Sicre, M.-A.; Cardoso, J. N.; Macedo, S. J.; Sci. Total Environ. 1999, 231, 1.

29. Wang, X.-C.; Sun, S.; Ma, H.-Q.; Liu, Y.; Mar. Pollut. Bull. 2006, 52, 129.

30. Mejanelle, L.; Laureillard, J.; Mar. Chem. 2008, 108, 59; Tolosa, I.;de Mora, S.; Sheikholeslami, M. R.; Villeneuve, J.-P.; Bartocci, J.; Cattini, C.; Mar. Pollut. Bull. 2004, 48, 44.
31. Medeiros, P. M.; Bícego, M. C.; Castelão, R. M.; Del Rosso, C.; Fillmann, G.; Zamboni, A. J.; Environ. Int. 2005, 31, 77.

32. Medeiros, P. M.; Bícego, M. C.; Mar. Pollut. Bull. 2004, 49, 761.

33. Bícego, M. C.; Taniguchi, S.; Yogui, G. T.; Montone, R. C.; Silva, D. A. M.; Lourenço, R. A.; Martins, C. C.; Sasaki, S. T.; Pellizari, V. H.; Weber, R. R.; Mar. Pollut. Bull. 2006, 52, 1804.

34. Meniconi, M. F. G.; Gabardo, I. T.; Carneiro, M. E. R.; Barbanti, S. M.; Silva, G. C.; Massone, C. G.; Environ. Forensics 2002, 3, 303.

35. Nishigima, F. N.; Weber, R. R.; Bícego, M. C.; Mar. Pollut. Bull. 2001, 42, 1064.

36. Bray, E. E.; Evans, E. D.; Geochim. Cosmochim. Acta 1961, $22,2$.

37. Aboul-Kassim, T. A. T.; Simoneit, B. R. T.; Mar. Chem. 1996, 54, 135.

38. Zhou, W.; Xie, S.; Meyers, P. A.; Zheng, Y.; Org. Geochem. 2005, 36, 1272 .

39. Belligotti, F. M.; Carreira, R. S.; Soares, M. L. G.; Geochim. Bras. 2007, 21,71 .

40. Jeng, W.-L.; Lin, S.; Kao, S.-J.; Deep-Sea Res. Part I: Topical Studies in Oceanography 2003, 50, 1179.

41. Bourbonniere, R. A.; Meyers, P. A.; Limnol. Oceanog. 1996, 41, 352.

42. Volkman, J. K.; Org. Geochem. 1986, 9, 83; Laureillard, J.; Saliot, A.; Mar. Chem. 1993, 43, 247.

43. Wang, X.-C.; Chen, R. F.; Berry, A.; Estuar. Coast. Shelf Sci. 2003, 58, 917; Gogou, A.; Bouloubassi, I.; Stephanou, E. G.; Mar. Chem. 2000, 68, 265.

44. Wang, Z.; Fingas, M.; Page, D. S.; J. Chromatogr., A 1999, 843, 369.

45. Readman, J. W.; Fillmann, G.; Tolosa, I.; Bartocci, J.; Villeneuve, J.-P.; Catinni, C.; Mee, L. D.; Mar. Pollut. Bull. 2002, 44, 48; Rushdi, A.; DouAbul, A.; Mohammed, S.; Simoneit, B.; Environ. Geol. 2006, 50, 857.

46. González-Oreja, J. A.; Saiz-Salinas, J.; Mar. Pollut. Bull. 1998, 36, 868.

47. Santos, E. S.; Carreira, R. S.;Knoppers, B. A.; Braz. J. Oceanogr. 2008 , $56,97$.

48. Cordeiro, L. G.; Carreira, R. S.; Wagener, A. L. R.; Org. Geochem. 2008 , 39, 1097.

49. Costa, R. L.;Carreira, R. S.; Braz. J. Oceanogr. 2005, 53, 157.

50. Martins, C. C.; Fillmann, G.; Montone, R. C.; J. Braz. Chem. Soc. 2007, $18,106$.

51. Martins, C. C.; Ferreira, J. A.; Taniguchi, S.; Mahiques, M. M.; Bícego, M. C.; Montone, R. C.; Mar. Pollut. Bull. 2008, 56, 1359.

52. Martins, C. C.; Gomes, F. B. A.; Ferreira, J. A.; Montone, R. C.; Quim. Nova 2008, 31, 1008.

53. Mater, L.; Alexandre, M. R.; Hansel, F. A.; Madureira, L. A. S.; J. Braz. Chem. Soc. 2004, 15, 725.

54. Kawakami, S. K., Dissertação de Mestrado, Universidade de São Paulo, Brasil, 1999.

55. Muniz, P.; Pires-Vanin, A. M. S.; Martins, C. C.; Montone, R. C.; Bicego, M. C.; Mar. Pollut. Bull. 2006, 52, 1098. 


\section{HIDROCARBONETOS E ESTEROIS COMO INDICADORES DE FONTES E DESTINO DE MATÉRIA ORGÂNICA EM SEDIMENTOS DA BAÍA DE SEPETIBA, RIO DE JANEIRO}

Renato S. Carreira*, Patrícia V. Ribeiro e Carlos E. M. Silva

Faculdade de Oceanografia, Universidade do Estado do Rio de Janeiro, 20550-013 Rio de Janeiro - RJ, Brasil

Cassia O. Farias

Departamento de Química, Pontifícia Universidade Católica do Rio de Janeiro, 22453-900 Rio de Janeiro - RJ, Brasil

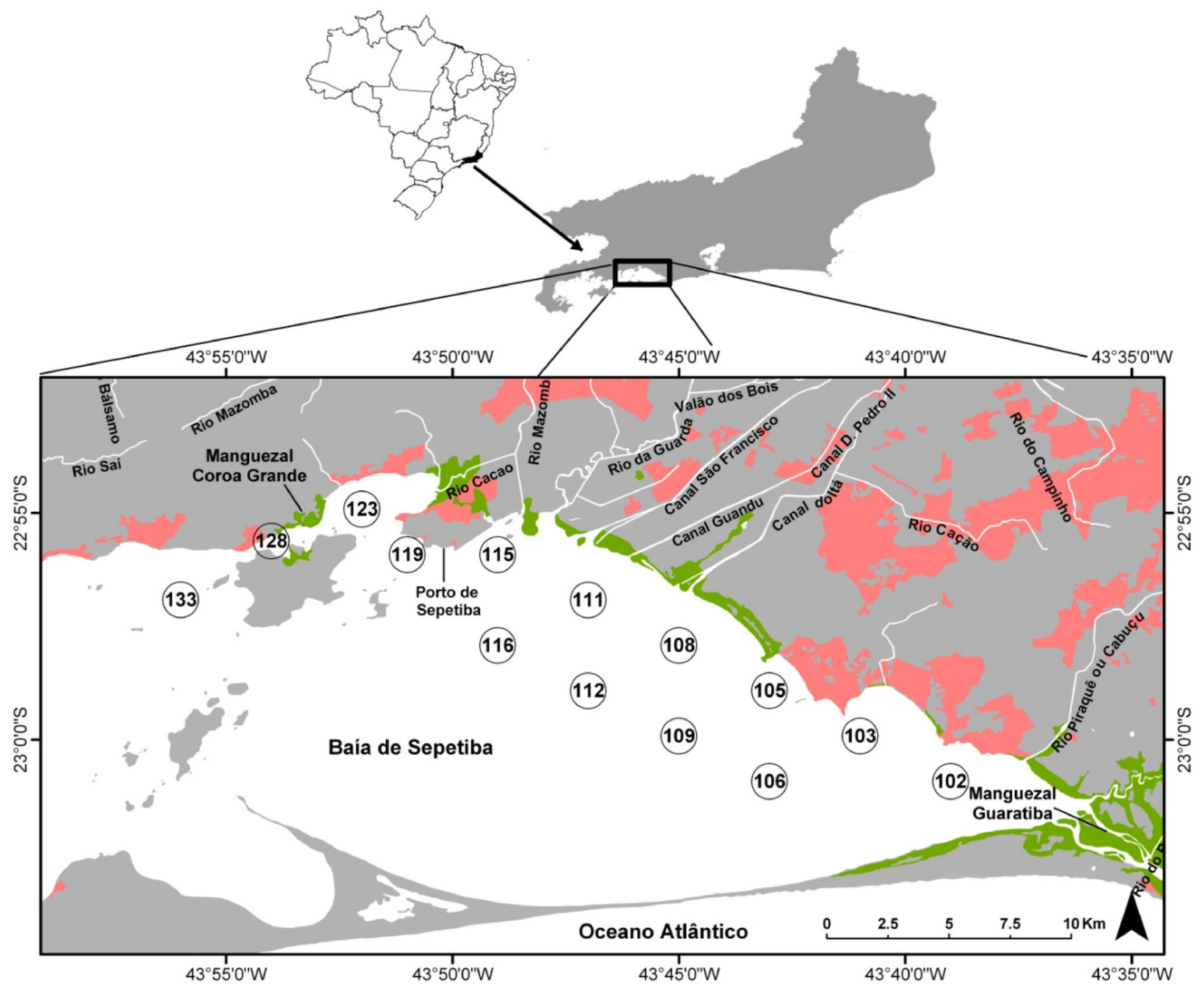

Figura 1S. Mapa da Baía de Sepetiba com os pontos de amostragem de sedimento. Áreas de ocupação urbana são representadas em vermelho e de manguezais, em verde 


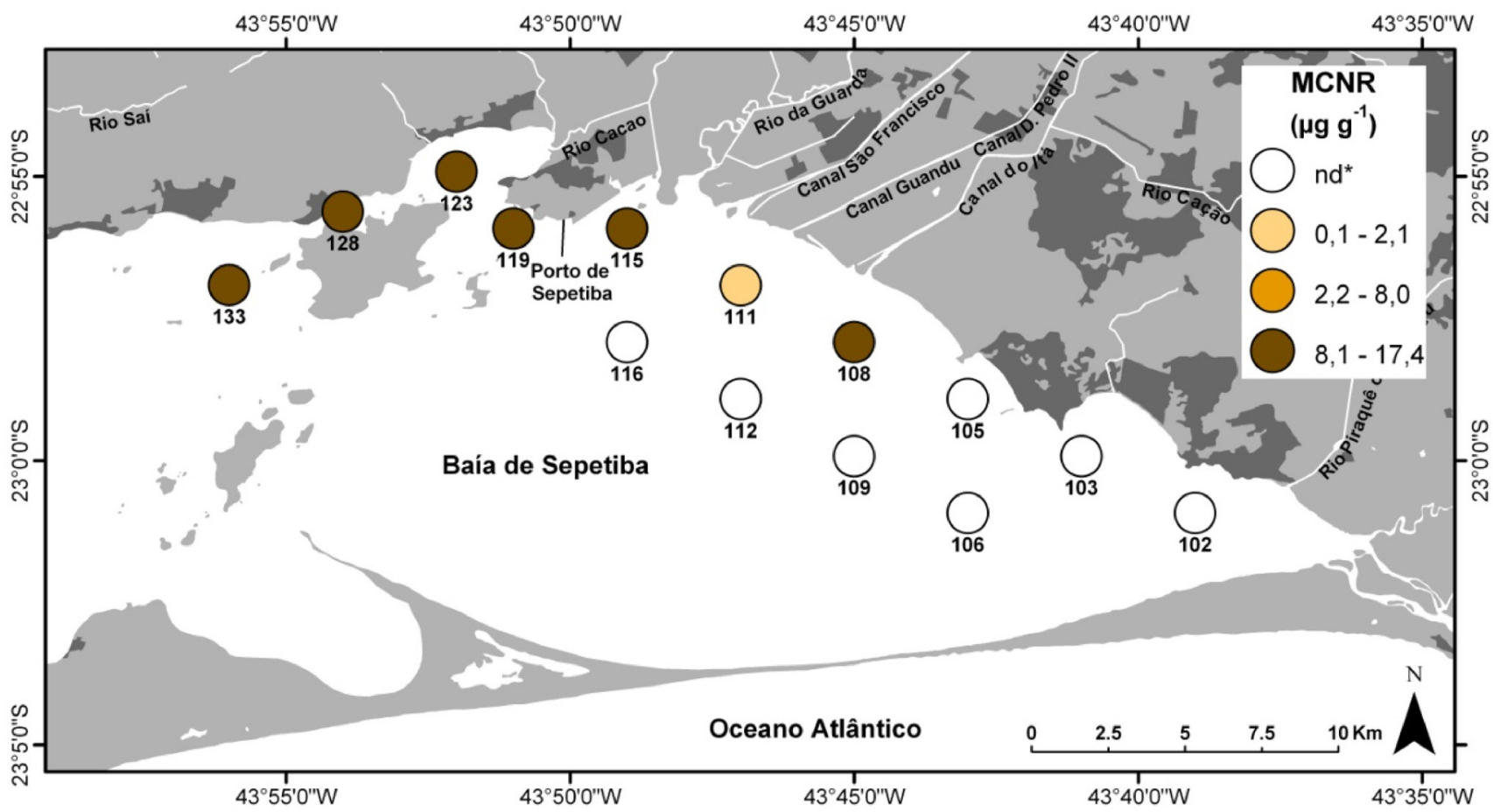

Figura 2S. Distribuição de mistura complexa não-resolvida (MCNR) nos sedimentos da Baía de Sepetiba. *nd = não detectado

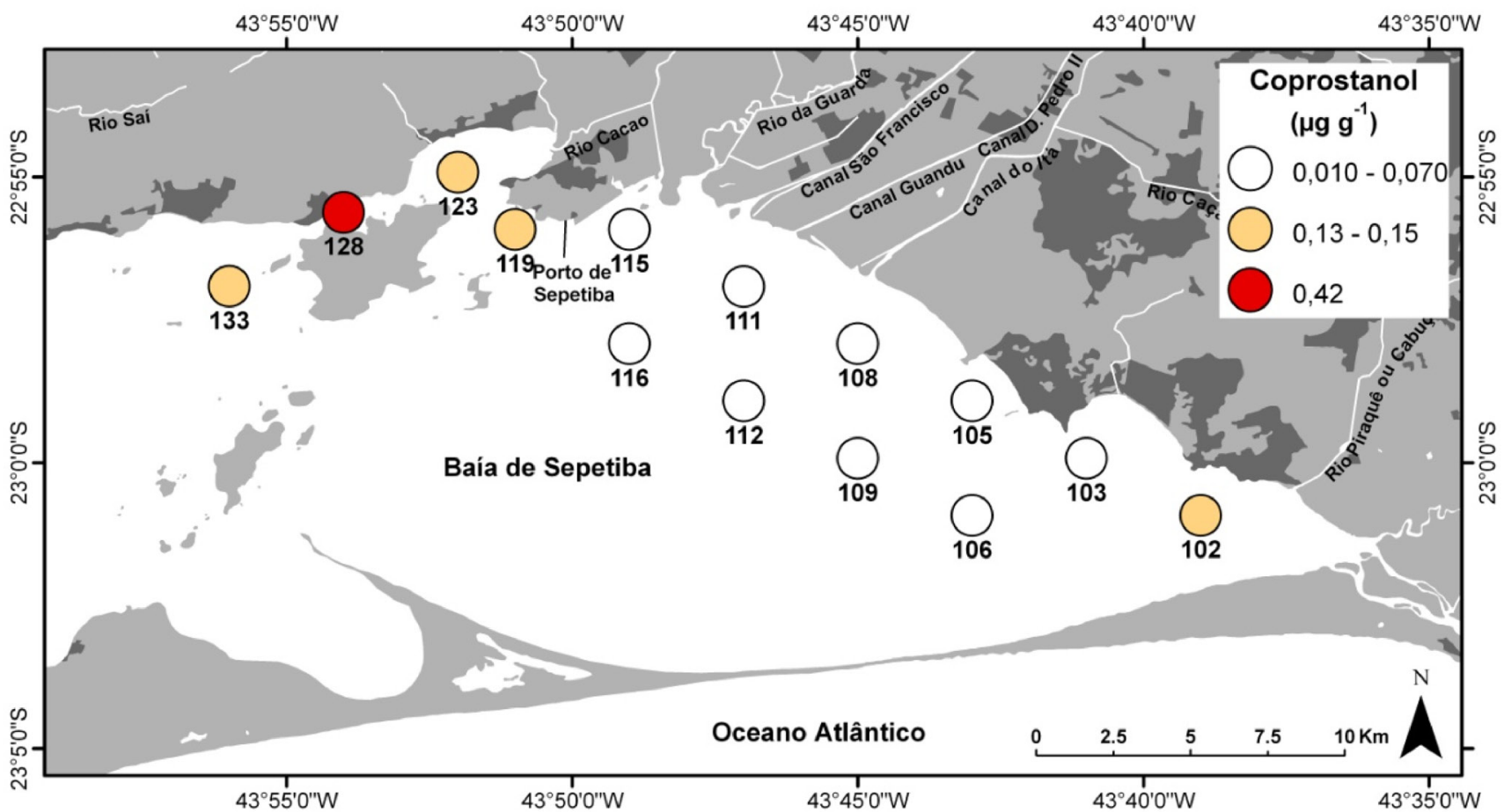

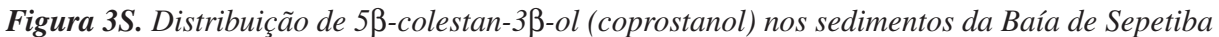

